CYSTIC FIBROSIS

\title{
Burkholderia cenocepacia and Burkholderia multivorans: influence on survival in cystic fibrosis
}

\author{
A M Jones, M E Dodd, J R W Govan, V Barcus, C J Doherty, J Morris, A K Webb
}

Thorax 2004;59:948-951. doi: 10.1136/thx.2003.017210

See end of article for authors' affiliations

Correspondence to:

Correspondence to:
Dr A M Jones, Manchester Adult Cystic Fibrosis

Centre, South Manchester

University Hospitals NHS

Trust, Wythenshawe

Hospital, Southmoor Road,

Manchester M23 9LT, UK;

andmarkj@hotmail.com

Received 28 October 2003

Accepted 9 July 2004

\begin{abstract}
Introduction: Burkholderia cepacia infection has been associated with a poor prognosis for patients with cystic fibrosis (CF). It is now recognised that organisms classified as $B$ cepacia comprise a number of distinct genomic species each known as a genomovar of the $B$ cepacia complex (BCC). The outcome of infection for $\mathrm{CF}$ patients with individual genomovars is unknown. The clinical outcome of infection with the two most commonly isolated genomovars ( $B$ cenocepacia and B multivorans) was studied at a specialist CF centre between 1982 and 2003.

Methods: The numbers of patients who progressed from initial to chronic infection were assessed. Control groups were created by matching patients with chronic $B C C$ infection by percentage forced expiratory volume in 1 second with patients with Pseudomonas aeruginosa infection. Outcome measures were survival time, deaths from "cepacia syndrome", rate of decline in spirometry and body mass index (BMI), and treatment requirements.

Results: Forty nine patients had an initial infection with either $B$ multivorans $(n=16)$ or $B$ cenocepacia $(n=33) ; 8 / 16$ and $31 / 33$, respectively, developed chronic infection $(p<0.001)$. Deaths from "cepacia syndrome" occurred in both BCC groups. Patients with B cenocepacia infection had a shorter survival than patients with $P$ aeruginosa infection $(p=0.01)$. There was no difference in survival between $C F$ patients infected with $B$ multivorans and $P$ aeruginosa. There were no observed differences in changes in spirometry and $B M I$ or treatment requirements between the $B C C$ groups and respective controls.

Conclusion: In CF, the genomovar status of $B C C$ may influence both the likelihood of progression from initial to chronic infection and the overall survival of the patients.
\end{abstract}

n the 1980s it became recognised that an organism known as Burkholderia cepacia was an opportunistic pathogen for individuals with cystic fibrosis (CF). ${ }^{1}$ Pulmonary infection with $B$ cepacia was associated with increased rates of morbidity and mortality. ${ }^{1-4}$ In a proportion of patients infection also caused a fulminating pneumonic illness with high temperatures and respiratory failure sometimes associated with septicaemia which became known as "cepacia syndrome". Furthermore, the emergence and spread of a number of epidemic strains presented cross-infection control problems for $\mathrm{CF}$ patients in the community and at CF centres. $^{5-7}$

Recent studies, however, have shown that organisms presumptively identified as B cepacia by conventional phenotypic tests constitute different genotypic species, each now known as a genomovar of the $B$ cepacia complex $(B C C) .{ }^{89}$ Although a considerable amount of work has been carried out in taxonomic analysis to define the different species of the $B C C$, the relevance of this classification to the CF clinician is unknown. The majority of $B C C$ isolates from $\mathrm{CF}$ centres belong either to $B$ multivorans ( $B C C$ genomovar II) or $B$ cenocepacia (BCC genomovar III). ${ }^{10}$ It is recognised that $\mathrm{CF}$ patients with $B C C$ infection can have a worse outcome following lung transplantation ${ }^{11}$ and this may depend on the genomovar status of the organism. ${ }^{12}{ }^{13}$ The overall morbidity and mortality for CF patients resulting from infection by different genomovars of the $B C C$ is, however, unknown. We now report the clinical outcome of patients with $B$ multivorans and $B$ cenocepacia infections at a specialist adult CF centre.

\section{METHODS}

Data were collected by a retrospective review of the case records of all CF patients infected with BCC at the Manchester Adult CF Centre. In order to prevent survivor bias, only patients who had become infected with $B C C$ since referral to the Adult Centre (1982-2003) were included in the study. For the purpose of this study, the date of acquisition of $B C C$ infection was defined as the date the organism was first isolated from sputum samples. $B C C$ infection was classified as transient if at least three consecutive cultures failed to yield the organism over a minimum period of 12 months.

Patients were grouped according to the results of genomovar typing of isolates identified by Rec-A PCR analysis. ${ }^{14}$ Typing of isolates by pulsed field gel electrophoresis (PFGE) was also performed to assess the clonality of isolates within each group. Cases in which $B C C$ strains have cross-infected CF patients already infected with other $B C C$ have been described. ${ }^{15}{ }^{16}$ We therefore reviewed previous typing results of patient isolates to see if they had changed their species and strain. For patients with $B$ multivorans infection we looked at the last isolate taken prior to death.

Using a research database, each patient who developed chronic BCC infection was individually matched with another CF patient who had been referred to the adult centre within the same 12 month period and had chronic Pseudomonas aeruginosa infection. Matching was by the percentage predicted forced expiratory volume in 1 second $\left(\% \mathrm{FEV}_{1}\right)$ at the time the $B C C$ species was first isolated. Patients with $B$ cenocepacia infection and patients with $B$ multivorans infection therefore each had their own control groups.

The age, $\% \mathrm{FEV}_{1}$, percentage predicted forced vital capacity (\%FVC), and body mass index (BMI) of the patients were calculated at acquisition and annually for up to 5 years after the acquisition date. A mean of the available values of a month either side of the acquisition date was taken. For each group of patients the number of deaths and time to death (in months) were recorded. For patients with $B C C$ infection the number of deaths from "cepacia syndrome" was also noted. 
Table 1 Baseline demographic data of patients with chronic BCC infection at acquisition and $P$ aeruginosa controls

\begin{tabular}{lllllll}
\hline & $\begin{array}{l}\text { B cenocepacia } \\
(\mathbf{n = 3 1 )}\end{array}$ & $\begin{array}{l}\mathbf{P} \text { aeruginosa } \\
(\mathbf{n = 3 1 )}\end{array}$ & $\mathbf{p}$ value & $\begin{array}{l}\text { B multivorans } \\
(\mathbf{n = 8})\end{array}$ & $\begin{array}{l}\boldsymbol{P} \text { aeruginosa } \\
(\mathbf{n = 8})\end{array}$ \\
\hline Age (years) & $22.0(4.7)$ & $23.4(5.2)$ & 0.14 & $21.8(4.8)$ & $21.7(4.1)$ & 0.97 \\
Sex (M/F) & $18 / 13$ & $14 / 17$ & 0.45 & $7 / 1$ & $6 / 2$ & 1.0 \\
\%FEV & $52.0(19.6)$ & $51.8(21.2)$ & 0.90 & $56.5(20.2)$ & $53.6(17.9)$ & 0.73 \\
\%FVC & $73.1(19.7)$ & $69.5(19.6)$ & 0.17 & $76.9(20.7)$ & $79.9(19.7)$ & 0.76 \\
BMI & $18.7(1.8)$ & $19.7(3.1)$ & 0.10 & $18.9(1.3)$ & $20.9(1.4)$ & 0.01 \\
\hline
\end{tabular}

$\mathrm{FEV}_{1}$, forced expiratory volume in 1 second; FVC, forced vital capacity; BMI, body mass index.

Results expressed as mean (SD).

Days spent on intravenous antibiotics each year, infective pulmonary exacerbations each year, and clinic visits each year were calculated for 5 years after the acquisition date. Days spent as an inpatient each year were also calculated, but hospital admissions for causes unrelated to respiratory exacerbations were excluded.

\section{Analysis of data}

Statistical analysis was performed using SPSS statistical package version 9.0 (SPSS Inc, Chicago, Illinois, USA). The two $B C C$ groups were compared with each other for number of patients who progressed from initial to chronic infection and number of deaths from "cepacia syndrome". Otherwise, the $B C C$ groups were compared with their respective control groups consisting of patients with $P$ aeruginosa infection. The percentage survival at 1 year and 5 years was calculated using a Kaplan-Meier plot and the groups were compared using the log rank test. Other comparisons were assessed by McNemar, Wilcoxon and paired $t$ tests.

\section{RESULTS}

Forty nine CF patients had become infected with $B$ multivorans $(\mathrm{n}=16)$ or $B$ cenocepacia $(\mathrm{n}=33)$ since referral to the Adult Centre. Another patient had chronic infection with a $B$ anthinia (BCC genomovar VIII) strain and a further six patients had developed infection with $B$ gladioli; these cases were not included in the present study. Ten patients had a transient infection: $8 / 16$ and $2 / 33$ with $B$ multivorans and $B$ cenocepacia, respectively $(\mathrm{p}<0.001)$. Interestingly, the two cases of transient infection with $B$ cenocepacia involved the notorious epidemic strain known as ET12.58

All $B$ multivorans isolates had unique PFGE typing profiles. In contrast, the majority (29/33) of isolates from patients with $B$ cenocepacia were identified by PFGE as the ET1 2 strain. None of the patients with $B$ multivorans infection developed superinfection with $B$ cenocepacia, but one patient with $B$ cenocepacia infection was found to have acquired a different strain of $B$ cenocepacia that replaced the original $B$ cenocepacia strain.

There were no significant differences between the $B C C$ groups and their respective controls with $P$ aeruginosa infection in age, sex, \% FEV 1 , and \%FVC at baseline, although patients with $B$ multivorans infection had a lower mean BMI than their controls (table 1). There were more deaths among the patients with $B$ cenocepacia infection than their respective

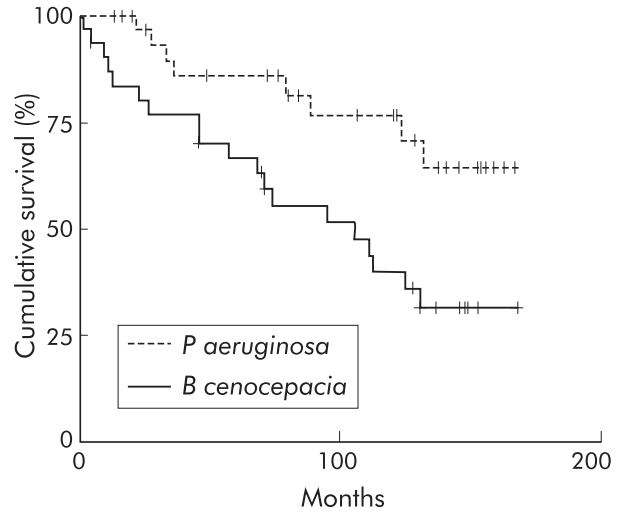

Figure 1 Survival of CF patients with chronic $B$ cenocepacia infection and matched controls with chronic $P$ aeruginosa infection $(n=31$ in each group).

controls, and patients with $B$ cenocepacia infection had a shorter survival time (table 2, fig 1). One patient with $B$ multivorans and two patients with $B$ cenocepacia infection died of "cepacia syndrome"; the times of death from initial infection were 22, 9, and 22 months, respectively.

Three patients with $B$ cenocepacia infection died within a year of developing infection. With the exception of survival times, there was insufficient clinical follow up data from the three patients, so all three patients and their three respective $P$ aeruginosa controls were excluded from the analysis of follow up clinical data. All other patients contributed to the data. There were no significant differences observed between either $B C C$ group and their respective controls in annual changes in $\% \mathrm{FEV}_{1}, \% \mathrm{FVC}$, and BMI (table 3 ). Patients with $B$ cenocepacia infection attended the outpatient department more times than their controls; otherwise, there were no significant differences between the BCC groups and their respective controls in annual number of respiratory exacerbations, inpatient days, and intravenous antibiotic treatment days (table 4 ).

\section{DISCUSSION}

This study examines whether infection with $B$ cenocepacia and $B$ multivorans respectively affect the clinical outcome of CF patients above that of $P$ aeruginosa, the most prevalent

Table 2 Survival of patients with $B C C$ infection and matched controls with $P$ aeruginosa infection

\begin{tabular}{llllll}
\hline & $\begin{array}{l}\text { B cenocepacia } \\
(\mathbf{n = 3 1 )}\end{array}$ & $\begin{array}{l}\mathbf{P} \text { aeruginosa } \\
(\mathbf{n = 3 1 )}\end{array}$ & P value & $\begin{array}{l}\text { B multivorans } \\
(\mathbf{n = 8})\end{array}$ & $\begin{array}{l}\boldsymbol{P} \text { aeruginosa } \\
(\mathbf{n = 8})\end{array}$ \\
\hline Deaths & 19 & 8 & - & 5 & 5 \\
Cepacia syndrome & 2 & - & - & 1 & - \\
1 year survival & $80.2 \%$ & $100 \%$ & & $100 \%$ & $100 \%$ \\
5 year survival & $66.6 \%$ & $85.3 \%$ & 0.01 & $75.0 \%$ & $75.0 \%$ \\
\hline
\end{tabular}


Table 3 Annual changes in spirometric parameters and body mass index (BMI) for CF patients with $B C C$ infection and matched controls with $P$ aeruginosa infection

\begin{tabular}{|c|c|c|c|c|c|c|c|c|}
\hline & $\begin{array}{l}\text { B cenocepacia } \\
(\mathrm{n}=27)\end{array}$ & $\begin{array}{l}P \text { aeruginosa } \\
(\mathrm{n}=27)\end{array}$ & $\begin{array}{l}\text { Estimated difference } \\
\text { P aeruginosa v } \\
\text { B cenocepacia } \\
(\mathrm{n}=27)\end{array}$ & $p$ value & $\begin{array}{l}\text { B multivorans } \\
(\mathrm{n}=8)\end{array}$ & $\begin{array}{l}P \text { aeruginosa } \\
(n=8)\end{array}$ & $\begin{array}{l}\text { Estimated difference } \\
\text { P aeruginosa v } \\
\text { B multivorans } \\
(\mathrm{n}=8)\end{array}$ & p value \\
\hline$\% \mathrm{FEV}_{1}{ }^{*}$ & $\begin{array}{l}-2.7 \% \\
(-47.1 \text { to }+13.0)\end{array}$ & $\begin{array}{l}-2.2 \% \\
(-16.6 \text { to }+2.0)\end{array}$ & $\begin{array}{l}-0.7 \% \\
(-14.4 \text { to }+30.1)\end{array}$ & 0.37 & $\begin{array}{l}-3.0 \% \\
(-15.3 \text { to }+1.4)\end{array}$ & $\begin{array}{l}-0.6 \% \\
(-9.9 \text { to }+2.0)\end{array}$ & $\begin{array}{l}-2.2 \% \\
(-33.6 \text { to }+38.7)\end{array}$ & 0.33 \\
\hline$\% \mathrm{FVC}^{*}$ & $\begin{array}{l}-1.7 \% \\
(-38.7 \text { to }+13)\end{array}$ & $\begin{array}{l}-2.8 \% \\
(-25.6 \text { to }+3.4)\end{array}$ & $\begin{array}{l}-5.9 \% \\
(-34.5 \text { to }+27.1)\end{array}$ & 0.40 & $\begin{array}{l}-3.3 \% \\
(-11.1 \text { to }+0.5)\end{array}$ & $\begin{array}{l}-0.6 \% \\
(-11.1 \text { to }+2.0)\end{array}$ & $\begin{array}{l}+9.3 \% \\
(-52.9 \text { to }+25.8)\end{array}$ & 0.33 \\
\hline BMI & $+0.7(1.5)$ & $+0.5(1.9)$ & $+0.9(-0.2$ to +2.0$)$ & 0.75 & $+0.6(0.9)$ & $+0.2(1.6)$ & $+2.0(+0.7$ to +3.3$)$ & 0.61 \\
\hline
\end{tabular}

Results expressed as mean (SD) or median (range) $)^{*}$ and estimated mean or median differences between the groups $(P$ aeruginosa $v B$ cenocepacia or $P$ aeruginosa $\checkmark$ B multivorans) and $95 \%$ confidence intervals or ranges.

pathogen for this group of patients. There was a greater overall number of deaths and significantly shortened survival for patients with $B$ cenocepacia infection in comparison with matched patients with $P$ aeruginosa infection. There was no difference in survival between patients infected with $B$ multivorans and $P$ aeruginosa, although the number of patients was small.

Transient infection with $B C C$ has been observed in $5-10 \%$ of cases, but the rates for individual genomovars are unclear. ${ }^{17}$ In our study the proportion of patients who had a transient infection was significantly greater with $B$ multivorans than $B$ cenocepacia $(\mathrm{p}<0.001)$. A recent Canadian study also described transient infection with $B$ multivorans in 10 of 19 patients. ${ }^{15}$ Of importance, two patients in our study also demonstrated transient infection with the ET12 strain of $B$ cenocepacia. The possibility of whether BCC infection may be transient is important for CF patients and carers, given the current stigma associated with BCC infection and its implications in terms of segregation policies. The UK CF Trust currently recommends a period of at least 1 year with at least three consecutive negative cultures before a patient is considered free of $B C C$ infection. ${ }^{17}$ In the meantime, the patient must be segregated from all other CF patients.

"Cepacia syndrome" is recognised as an almost untreatable clinical condition. In the present study there were cases of cepacia syndrome in association with both $B$ cenocepacia and $B$ multivorans.

Spirometry and nutritional status are known predictors of survival for individuals with CF. The effects of $B$ cepacia infection on lung function and its relationship to the increased mortality associated with the organism for individuals with CF are complex. Mundi et $a l^{3}$ found that $B$ cepacia infection caused an accelerated decline in pulmonary function and an increased mortality, although studies by Isles et $a l^{1}$ and Frangolias et $a l^{2}$ did not find any significant difference in decline in lung function but did demonstrate a survival disadvantage in comparison with non-infected CF patients. In the present study patients with $B$ cenocepacia had a decreased survival but without significant change in spirometry compared with their control group. Mean BMI increased in all patient groups in the present study. This may seem somewhat unusual; however, the increase in BMI over time is a reflection of the overall trend in nutritional status for all CF patients at our centre. There was no difference in weight loss between $B$ cepacia infected and non-infected CF patients in a recent study. ${ }^{2}$

Previous studies have shown an increase in the use of hospital services and treatment requirements for CF patients following $B$ cepacia infection in terms of a significant rise in the number of inpatient days, intravenous antibiotic days, and outpatient visits. ${ }^{2-4}$ In the present study, patients with $B$ cenocepacia infection were found to have a greater number of clinic visits than patients with $P$ aeruginosa infection. However, the $B C C$ groups did not significantly differ in the number of infective exacerbations, requirement for inpatient treatment, and intravenous antibiotics in comparison with their control groups. The factors influencing the clinical course of a patient following acquisition of $B C C$ infection are clearly complex and probably influenced by multiple factors, including host response to the pathogen. As evidence for the importance of host factors, an early study showed that patients infected with the same ET12 strain of B cenocepacia displayed marked differences in their clinical course. ${ }^{5}$

The majority of BCC infections at the Manchester Adult CF Centre are with $B$ multivorans $(30.6 \%)$ or $B$ cenocepacia $(67.3 \%)$. While each patient's $B$ multivorans isolate is a unique strain, the majority $(87.9 \%)$ of $B$ cenocepacia isolates belong to a single epidemic strain (the ET12 strain). Most highly transmissible strains of $B C C$ are $B$ cenocepacia, ${ }^{10} 18$ although $B$ multivorans strains have been associated with epidemic spread. ${ }^{10}{ }^{19}$ The introduction of strict segregation policies over the last decade will probably influence the proportion of new infections belonging to the different genomovars.

Mahenthiralingam et al reported a higher mortality among CF patients with $B$ cenocepacia in Vancouver than patients

Table 4 Annual treatment requirements between CF patients with $B C C$ infection and matched controls with $P$ aeruginosa infection

\begin{tabular}{|c|c|c|c|c|c|c|c|c|}
\hline & $\begin{array}{l}\text { B cenocepacia } \\
(n=27)\end{array}$ & $\begin{array}{l}P \text { aeruginosa } \\
(n=27)\end{array}$ & $\begin{array}{l}\text { Estimated difference } \\
P \text { aeruginosa } v \\
\text { B cenocepacia }(n=27)\end{array}$ & p value & $\begin{array}{l}\text { B multivorans } \\
(n=8)\end{array}$ & $\begin{array}{l}P \text { aeruginosa } \\
(n=8)\end{array}$ & $\begin{array}{l}\text { Estimated difference } \\
P \text { aeruginosa } v B \\
\text { multivorans } \\
(n=8)\end{array}$ & $\mathrm{p}$ value \\
\hline Exacerbations & $5.5(2.4)$ & $5.2(3.2)$ & $-0.3(-1.8$ to +1.2$)$ & 0.66 & $5.5(4.2)$ & $4.9(3.4)$ & $-0.5(-4.8$ to +3.7$)$ & 0.77 \\
\hline Clinic visits & $12.6(5.3)$ & $9.2(4.8)$ & $-3.5(-5.9$ to -1.1$)$ & $<0.01$ & $12.1(6.7)$ & $11.6(7.8)$ & $-0.5(-8.6$ to +7.5$)$ & 0.88 \\
\hline Inpatient days* & $\begin{array}{l}27.8 \\
(0 \text { to } 91.4)\end{array}$ & $\begin{array}{l}17.4 \\
(0 \text { to } 247.0)\end{array}$ & $\begin{array}{l}-2.6 \\
(-91.4 \text { to }+214.2)\end{array}$ & 0.81 & $\begin{array}{l}12.2 \\
(1.4 \text { to } 118.0)\end{array}$ & $\begin{array}{l}5.5 \\
(0 \text { to } 61.5)\end{array}$ & $\begin{array}{l}-7.2 \\
(-115.5 \text { to }+53.7)\end{array}$ & 0.48 \\
\hline $\begin{array}{l}\text { Intravenous } \\
\text { antibiotics* }\end{array}$ & $\begin{array}{l}45.2 \\
(0 \text { to } 147.0)\end{array}$ & $\begin{array}{l}44.0 \\
(0 \text { to } 265.0)\end{array}$ & $\begin{array}{l}0 \\
(-126.4 \text { to }+226.4)\end{array}$ & 0.75 & $\begin{array}{l}19.6 \\
(9.0 \text { to } 171.0)\end{array}$ & $\begin{array}{l}15.3 \\
(0 \text { to } 136.0)\end{array}$ & $\begin{array}{l}-10.6 \\
(-154.0 \text { to }+111.5)\end{array}$ & 0.40 \\
\hline
\end{tabular}

Results expressed as mean (SD) or median (range) $)^{*}$ and estimated mean or median differences between the groups ( $P$ aeruginosa $v B$ cenocepacia or $P$ aeruginosa $\checkmark B$ multivorans) and $95 \%$ confidence intervals or ranges. 
with $B$ multivorans. ${ }^{15}$ However, there were significant age differences between the two groups. The present study has compared patients with Burkholderia species with matched controls with $P$ aeruginosa using $\% \mathrm{FEV}_{1}$. It should be acknowledged that, as the survival analysis does not allow for the matching, the effect estimates may be conservative.

A limitation of this study is the small numbers of patients, particularly those with $B$ multivorans infection. A further limitation is that the data and conclusions have been heavily influenced by the presence of a single highly transmissible $B$ cenocepacia strain. In the study by Mahenthiralingam et al all the patients with $B$ cenocepacia also had transmissible strains. The only other published studies of outcome of BCC infection in CF have studied patients following transplantation. ${ }^{12} 13$ Chapparo et al $^{11}$ found a poorer outcome for CF patients with $B$ cepacia infection following lung transplantation, and De Soyza $\mathrm{et} \mathrm{al}^{12}$ reported a worse outcome following transplantation with B cenocepacia than with other BCC. Finally, Aris et a ${ }^{13}$ found that decreased survival after transplantation was linked to genomovar status irrespective of strain type, with a worse outcome for CF patients with $B$ cenocepacia infection, all of whom harboured unique genotypic strains. Further studies of the clinical effects of BCC infection in CF patients are required.

Replacement of existing $B C C$ by the $B$ cenocepacia strain ET12 has been reported. ${ }^{15}{ }^{16}$ Our study reviewed the clinical outcome between groups of patients with infection by strains that differ in their genomovar status. None of the patients in the present study are known to have died as a result of crossinfection by a different genomovar of $B C C$, although one patient did acquire a different strain but of the same genomovar. Current UK guidelines advocate a policy of segregation of CF patients with $B C C$ infection irrespective of genomovar status. ${ }^{17}$

This study has shown that the rate of transient infection and clinical outcome, including patient survival, for $B C C$ infection in CF may be influenced by genomovar status, and perhaps by individual strains within genomovars.

\section{Authors' affiliations}

A M Jones, M E Dodd, A K Webb, Manchester Adult Cystic Fibrosis Centre, South Manchester University Hospitals NHS Trust, Wythenshawe Hospital, Manchester M23 9LT, UK

J R W Govan, V Barcus, C J Doherty, Medical Microbiology, University of Edinburgh, Edinburgh EH8 9AG, UK

J Morris, Department of Medical Statistics, South Manchester University Hospitals NHS Trust, Wythenshawe Hospital, Manchester M23 9LT, UK This study was unfunded.

\section{REFERENCES}

1 Isles A, Maclusky I, Corey M, et al. Pseudomonas cepacia infection in cystic fibrosis: an emerging problem. J Pediatr 1984;104:206-10.

2 Frangolias DD, Mahenthiralingam E, Rae S, et al. Burkholderia cepacia in cystic fibrosis. Variable disease course. Am J Respir Crit Care Med 1999;160:1572-7.

3 Muhdi K, Edenborough FP, Gumery L, et al. Outcome for patients colonised with Burkholderia cepacia in a Birmingham adult cystic fibrosis clinic and the end of an epidemic. Thorax 1996;51:374-7.

4 Tablan OC, Martone WJ, Doershuk CF, et al. Colonization of the respiratory tract with Pseudomonas cepacia in cystic fibrosis. Risk factors and outcomes. Chest 1987;91:527-32.

5 Govan JR, Brown PH, Maddison J, et al. Evidence for transmission of Pseudomonas cepacia by social contact in cystic fibrosis. Lancet 1993;342:15-9.

6 Lipuma JJ, Dasen SE, Nielson DW, et al. Person-to-person transmission of Pseudomonas cepacia between patients with cystic fibrosis. Lancet 1990;336:1094-6.

7 Johnson WM, Tyler SD, Rozee KR. Linkage analysis of geographic and clinical clusters in Pseudomonas cepacia infections by multilocus enzyme electrophoresis and ribotyping. J Clin Microbiol 1994;32:924-30.

8 Mahenthiralingam E, Coenye T, Chung JW, et al. Diagnostically and experimentally useful panel of strains from the Burkholderia cepacia complex. J Clin Microbiol 2000;38:910-3.

9 Vandamme P, Holmes B, Vancanneyt $M$, et al. Occurrence of multiple genomovars of Burkholderia cepacia in cystic fibrosis patients and proposal of Burkholderia multivorans sp. nov. Int J Syst Bacteriol 1997; 47:1 188-200.

10 LiPuma JJ, Spilker T, Gill LH, et al. Disproportionate distribution of Burkholderia cepacia complex species and transmissibility markers in cystic fibrosis. Am J Respir Crit Care Med 2001;164:92-6.

11 Chaparro C, Maurer J, Gutierrez C, et al. Infection with Burkholderia cepacia in cystic fibrosis: outcome following lung transplantation. Am J Respir Crit Care Med 2001;163:43-8.

12 De Soyza A, McDowell A, Archer L, et al. Burkholderia cepacia complex genomovars and pulmonary transplantation outcomes in patients with cystic fibrosis. Lancet 2001;358:1780-1.

13 Aris RM, Routh JC, LiPuma JJ, et al. Lung transplantation for cystic fibrosis patients with Burkholderia cepacia complex. Am J Respir Crit Care Med 2001;164:2102-2106.

14 Mahenthiralingam E, Bischof J, Byrne SK, et al. DNA-Based diagnostic approaches for identification of Burkholderia cepacia complex, Burkholderia vietnamiensis, Burkholderia multivorans, Burkholderia stabilis, and Burkholderia cepacia genomovars I and III. J Clin Microbiol 2000;38:3165-73.

15 Mahenthiralingam E, Vandamme $P$, Campbell ME, et al. Infection with Burkholderia cepacia complex genomovars in patients with cystic fibrosis: virulent transmissible strains of genomovar III can replace Burkholderia multivorans. Clin Infect Dis $2001 ; 33: 1469-75$.

16 Ledson MJ, Gallagher MJ, Corkill JE, et al. Cross infection between cystic fibrosis patients colonised with Burkholderia cepacia. Thorax 1998;53:432-6.

17 Cystic Fibrosis Trust infection Control Group. A statement on Burkholderia cepacia. London: Cystic Fibrosis Trust, 1999.

18 Mahenthiralingam E, Simpson DA, Speert DP. Identification and characterization of a novel DNA marker associated with epidemic Burkholderia cepacia strains recovered from patients with cystic fibrosis. J Clin Microbiol 1997;35:808-16.

19 Segonds C, Heulin T, Marty G. Differentiation of Burkholderia species by PCRrestriction fragment length polymorphism analysis of the $16 \mathrm{~S}$ rRna gene and application to cystic fibrosis isolates. J Clin Microbiol 1999;37:2201-8. 